Ann. Biol. anim. Bioch. Biophys., 1979, 19 (3 B), 723-728.

\title{
Synthèse d'acide lactique par le cæcum de rat au cours du transfert des acides gras volatils
}

\author{
par P. MOTTAZ, J. F. WORBE
}

Laboratoire de Physiologie méfabolique, Université Claude Bernard Lyon I 43, Boulevard du 11 novembre 191869621 Villeurbonne.

Summary. Rat caecum lactate formation during volatile fatty acid transit.

Lactate synthesis was studied in vitro using the everted sacs of the rat caecum. The sacs were filled with $2 \mathrm{ml}$ of saline medium and then shaken $1 \mathrm{hr}$ at $38^{\circ} \mathrm{C}$ in flasks containing $25 \mathrm{ml}$ of the same medium. Krebs-Ringer bicarbonate incubation medium, saturated with 95 p. $100 \mathrm{O}_{2}$, was used with no substrate or with one of the following : (i) propionate (25 $\mathrm{mM}$ or $100 \mathrm{mM}$ ) alone, (ii) a mixture of acetic $(60 \mathrm{mM})$, propionic $(25 \mathrm{mM})$ and butyric $(15 \mathrm{mM})$ acids, (iii) glucose $(27 \mathrm{mM})$ alone. After incubation, the flask contents were homogenized and centrifuged, and the L-lactate and D-glucose enzymatically determined on protein-free supernatants. Propionate increased lactate production; acetate and butyrate had no effect. When glucose was utilized as a substrate, L-lactate formation was significantly greater than with propionate. The rat caecum converted less glucose than the jejunum. L-lactate formation in vivo or from endogenous substrate is discussed.

\section{Introduction.}

Une véritable symbiose entre l'hôte ef les microorganismes se situe dans le rumen des herbivores ou dans la dernière partie du tube digestif des espèces monogastriques. Les glucides sont dégradés par la microflore et transformés en composés labiles absorbables, en particulier en acides gras volatils, qui participeront à la gluconéogenèse (Leng, 1970 ; Leng, Steel et Luick, 1969).

Chez les ruminants, une quantité non négligeable d'acides gras volatils est métabolisée au cours de l'absorption par la paroi du rumen. L'acide butyrique se transforme en partie en corps cétoniques (Spahr, Kessler et Flipser, 1965 ; Stevens et Stettler, 1967). L'acide propionique peut être converti en acide lactique in vivo et in vitro par l'épithélium du rumen de Mouton (Pennington et Sutherland, 1956) ou de Bœuf (Weekes, 1974). Cela peut concerner 70 p. 100 du propionate intra ruminal du Mouton (Leng, Steel et Luick, 1967) mais seulement 2,3 p. 100 du propionate absorbé par le rumen chez le Veau (Weigand, Young et McGillard, 1972). Stevens ef Stettler (1967), ont montré que des fragments isolés d'épithélium de rumen de Bœuf utilisent le butyrate proportionnellement davantage que le propionate, lui même davantage que l'acétate, et donnent des corps cétoniques et d'autres molécules. 
Le métabolisme des acides gras volatils par la paroi du cæcum et du côlon a été moins étudié. Il est beaucoup moins important que celui qui est réalisé par le rumen. La paroi cæcale du Lapin (Henning et Hird, 1972) ou du Cheval (Stevens, 1970) transforment un peu de butyrate en corps cétoniques. Nous avons montré que la paroi cæcale du Rat métabolise davantage l'acide butyrique que les acides acétique et propionique (Worbe et Mottaz, 1970 ; Mottaz et Worbe, 1977).

La muqueuse de l'intestin grêle est le siège, au cours de l'absorption du glucose, d'une importante glycolyse, tant aérobie qu'anaérobie, qui aboutit à une production d'acide lactique d'inégale importance suivant les espèces animales. Elle est particulièrement élevée chez le Rat (Spencer et Knox, 1960 ; Clark et Sherratt, 1967 ; Worbe, Mottaz et Sadeghi, 1969).

Toutes ces observations nous ont amenés à étudier la production d'acide lactique au cours du transfert des acides gras volatils dans la paroi cæcale in vitro, et à la comparer à ce qui a été observé au niveau du rumen.

\section{Matériel et méthodes.}

Nous avons utilisé des Rats mâles, de souche Wistar, pesant $350 \mathrm{~g}$ et se nourrissant ad libitum avec un aliment standard jusqu'au moment du sacrifice. Le cæcum est prélevé, lavé avec du sérum physiologique, ligaturé au niveau de la valvule iléocæcale, puis retourné suivant la technique de Wilson et Wiseman (1954). Deux ml de liquide physiologique sont injectés dans le sac par l'extrémité aborale sur laquelle une ligature est serrée. L'organe est alors introduit dans un erlenmayer renfermant $25 \mathrm{ml}$ de liquide physiologique identique à celui qui a été introduit dans le sac et agité à $38^{\circ} \mathrm{C}$.

Les milieux utilisés sont préparés à partir d'une solution de Ringer bicarbonatée selon Krebs et Henseleit (1932), équilibrée pendant toute la durée de l'incubation par un mélange d'oxygène ( 95 p. 100) et de gaz carbonique ( 5 p. 100). Ils contiennent soit de l'acide propionique ( $25 \mathrm{mM}$ ou $100 \mathrm{mM})$, soit un mélange d'acide acétique $(60 \mathrm{mM})$, d'acide propionique $(25 \mathrm{mM})$ et d'acide butyrique $(15 \mathrm{mM})$, soit du glucose $(27 \mathrm{mM})$. Leur pH est égal à 7,4. Les sels d'acides gras sont donc entièrement ionisés.

Après $1 \mathrm{~h}$ d'incubation, le tissu cæcal est broyé dans les milieux qu'il séparait, et centrifugé. Le L-lactate ef le glucose sont dosés par des techniques enzymatiques dans le surnageant (Gutmann et Wahlefeld, 1974 ; Huggett et Nixon, 1957). Ces analyses permettent de déterminer les quantités de lactate présent et de glucose restant à la fin de l'incubation.

Pour savoir si la paroi cæcale apporte de l'acide lactique au début de l'incubation, nous avons prélevé, lavé et retourné le cæcum de rats qui venaient d'être sacrifiés. L'organe a été broyé et centrifugé. L'acide lactique a été dosé dans le surnageant.

L'acide lactique a aussi été dosé dans les matières se trouvant dans la lumière du cæcum au moment du sacrifice des animaux.

\section{Résultats.}

Les matières cæcales de 9 Rats, se nourrissant ad libitum jusqu'au moment de l'intervention, ont été analysées. Elles contiennent très peu d'acide lactique : 
$1,75 \pm 0,27 \mu \mathrm{mol}$ p. $\mathrm{ml}$ de liquide. C'est très comparable aux valeurs relevées dans les contenus de rumen (Annisson et Lewis, 1959).

L'acide lactique a été dosé dans la paroi cæcale de 9 Rats. Nous avons trouvé $4,28 \pm 0,56 \mu \mathrm{mol} p . g$ de tissu frais. Cette valeur représente la quantité d'acide lactique que l'organe apporte au temps zéro de nos expériences d'incubation.

Les résultats obtenus à la suite d'une heure d'incubation du cæcum éversé, placé entre un milieu muqueux ef un milieu séreux contenant des acides gras volatils ou du glucose, sont réunis dans le tableau 1 . On voit que la paroi du cæcum produit un peu d'acide lactique, même en l'absence de substrat exogène. Elle produit plus d'acide lactique si on lui fournit de l'acide propionique; la quantité obtenue dans la deuxième série d'expérieness est supérieure à celle obtenue dans la première série et la différence est statistiquement significative $(P<0,01)$. L'augmentation du laux d'acide propionique (série 3) ne modifie pratiquement pas la synthèse d'acide lactique : $100 \mathrm{mmol}$ p. I entraînent une formation d'acide lactique à peine supérieure à ce qu'entraîne $25 \mathrm{mmol}$ p. I, la différence n'ayant pas de valeur significative. La présence d'acide acétique et d'acide butyrique (série 4) n'augmente pas non plus la quantité d'acide lactique formé. Par contre, si le substrat exogène est du glucose, la paroi cæcale l'utilise pour produire plus d'acide lactique $(P<0,01)$ qu'à partir de l'acide propionique.

TABLEAU 1

Acide lactique formé ef glucose consommé par le cæcum de rat.

Les résultats sont rapportés à $1 \mathrm{~g}$ de tissu frais incubé pendant $1 \mathrm{~h}$

\begin{tabular}{ccccc}
\hline $\begin{array}{c}\text { Série } \\
\text { No }\end{array}$ & $\begin{array}{c}\text { Nombre } \\
\text { d'animaux }\end{array}$ & \multicolumn{1}{c}{ Substrat utilisé } & $\begin{array}{c}\text { Acide lactique dosé } \\
(\mu \mathrm{mol} \mathrm{p} . \mathrm{g})\end{array}$ & $\begin{array}{c}\text { Glucose consommé } \\
(\mu \mathrm{mol} \mathrm{p} . \mathrm{g})\end{array}$ \\
\hline 1 & 10 & Néant & $9,25 \pm 1,03$ \\
\hline 2 & 9 & Acide propionique $25 \mathrm{mM}$ & $13,22 \pm 0,91$ \\
\hline 3 & 10 & Acide propionique $100 \mathrm{mM}$ & $14,10 \pm 0,40$ \\
\hline 4 & 13 & $\begin{array}{l}\text { Acide acétique } 60 \mathrm{mM} \\
\text { Acide propionique } 25 \mathrm{mM}\end{array}$ & $13,68 \pm 0,67$ \\
\hline 5 & Acide butyrique $15 \mathrm{mM}$ & $18,25 \pm 3,01$ & $30,09 \pm 3,39$ \\
\hline
\end{tabular}

\section{Discussion.}

Nos résultats mettent nettement en évidence une activité métabolique du cæcum entraînant la synthèse d'acide lactique aussi bien in vivo qu'in vitro. In vivo, ce métabolite n'est pas libéré dans le tube digestif car les matières intraluminales en contiennent une très petite quantité qui est, sans doute, produite par la flore. L'organe ne libère pas immédiatement dans la circulation l'acide lactique qu'il produit. On le trouve, en effet, dans l'ensemble des tissus muqueux et musculaires à une concentration 2,5 fois supérieure à celle du contenu cæcal. Il est vraisemblable que la concentration est plus élevée dans l'épithélium. 
De l'acide lactique est apporté au début de l'expérience par le cæcum isolé. La quantité dosée après $1 \mathrm{~h}$ d'incubation ne représente donc pas uniquement le résultat des actions enzymatiques qui ont eu lieu. L'acide lactique étant un métabolite labile, il n'est pas possible d'affirmer que la quantité produite est la différence entre la quantité dosée à la fin de l'expérience et la quantité contenue dans le cæcum au temps zéro.

Nos résultats mettent en évidence que l'acide propionique augmente la quantité d'acide lactique formée par le tissu. Les deux autres acides gras n'ont pas cet effet. On peut penser que le succinyl-coenzyme A qui résulte du métabolisme de l'acide propionique s'incorpore au cycle de Krebs (Kaziro et Ochoa, 1964). La malate déshydrogénase (E. C. 1.1.1.40 L-Malate : NADP oxydo-réductase, decarboxylating) ef la phosphoénolpyruvate-carboxykinase (E. C. 4.1.1.32 GTP : oxaloacétate carboxylyase, transphosphorylating) permettent sa transformation en lactate (Smith et Osborne-White, 1971). In vivo, le foie permet à cette voie d'être glucoformatrice.

Le tissu cæcal peut aussi produire de l'acide lactique à partir du glucose. L'organe est donc le siège d'une glycolyse anaérobie même s'il est oxygéné (Worbe, Mottaz et Richardot, 1971). Cette activité est très inférieure à celle de la paroi du jéjunum de Rat placé dans les mêmes conditions. Darmenton (1969) a montré que des sacs de jéjunum incubés dans un milieu contenant du glucose $27 \mathrm{mM}$ produisaient $152 \mu \mathrm{mol}$ d'acide lactique p. g de tissu frais. Srivastava ef Hübscher (1966) ont étudié ce métabolisme et ses variations et montré que l'hexokinase peut être considérée comme un de ses facteurs limitants. L'importante formation de lactate à partir des préparations d'intestin de Rat a été attribuée à une absence de l'effet Pasteur de ce tissu (Sherratt, 1968 ; Tejwani ef al., 1974). Leese et Bronk (1975) ont montré que l'incubation des coupes d'intestin présente deux phases, une phase très rapide, d'une minute environ, correspondant à une libération importante de lactate, suivie d'une phase où cette libération diminue montrant un effet Pasteur positif. Hanson ef Parsons (1976) suggèrent que le lactate est formé dans une partie du tissu facilement accessible au glucose de la lumière intestinale, mais peu au glucose du sang circulant.

L'acide lactique peut aussi être produit à partir de substrats endogènes. En l'absence de substrat exogène, le tissu et les milieux contiennent plus d'acide lactique à la fin de l'incubation qu'au début. L'acide propionique n'est sans doute pas concerné car le cæcum n'en contient que $0,69 \mu \mathrm{mol}$ p. g au temps zéro (Mottaz, 1976). Le glucose provenant de la glycogénolyse qui aurait lieu au cours de l'incubation est probablement à l'origine de l'acide lactique.

Nous avons montré (Worbe, Mottaz et Richardot, 1971 ; Mottaz et Worbe, 1977) que l'activité absorbante de l'épithélium du cæcum de Rat est très limitée pour le glucose, comme pour les acides gras volatils. II est intéressant de constater que ce phénomène est lié à un métabolisme énergétique inférieur à celui de l'intestin grêle. On peut cependant conclure de ce travail que l'absorption de ces molécules s'accompagne d'une synthèse d'acide lactique. Dans les conditions physiologiques de digestion, c'est essentiellement l'acide propionique qui est à l'origine de cette synthèse. 


\section{Références}

ANNISON E. F., LEWIS D., 1959. Carbohydrate and volatile fatty acid metabolism, 59-91. In ANNISON E. F., LEWIS D., Mefabolism in the rumen. Methuen and Cie Ltd., London, John Wiley and Sons, New-York.

CLARK N., SHERRATT H. S. A., 1967. Glycolysis and oxydation in preparations from small intestinal mucosa of four species. Comp. Biochem. Physiol., 20, 223-243.

DARMENTON P., 1969. Absorption intestinale des glucides. Interaction glucose-galactose ef glucoselactose chez le rat. Doct. Spécial., Lyon, 133 pp.

GUTMANN I., WAHLEFELD A. W., 1974. L- $(+)$-Lactate. Determination with lactate dehydrogenase and NAD, 1464-1491. In BERGMEYER H. U., Methods of enzymatic analysis, vol. 3, Verlag Chemie Weiheim, Acad. Press, New-York.

HANSON P. J., PARSONS D. S., 1976. The utilization of glucose and profuction of lactate by in vitro preparations of rat small intestine. Effects of vascular perfusion. J. Physiol. Lond. 255, 775-795.

HENNING S. J., HIRD F. J. R., 1972. Transport of acetate and butyrate in the hindgut of rabbits. Biochem J., 130, 791-796.

HUGGETT A. S. C., NIXON D. A., 1957. Use of glucose oxydase, peroxyJase and O-dianisidine in determination of blood and urinary glucose. Lancet, 273, 368-370.

KAZIRO Y., OCHOA S., 1964. The metabolism of propionic acid. Adv. Enzymol., 26, 283-378.

KREBS H. A., HENSELEIT K., 1932. Untersuchungen uber die Harnstoffbildung im Tierkörper. $Z$. Physiol. Chem., 210, 33-66.

LEESE H. J., BRONK J. R., 1975. Lactate formation by rat small intestine in vitro. Biochim. biophys. Acta, 404, 40-48.

LENG R. A., 1970. Glucose synthesis in ruminants. Adv. vet. Sci., 14, 209-260.

LENG R. A., STEEL J. W., LUICK J. R., 1967. Contribution of propionate to glucose synthesis in sheep. Biochem. J., 103, 785-790.

MOTTAZ P., 1976. Absorption des acides gras volatils par le cæcum de rat. Doct. Spécial., Lyon, 162 pp.

MOTTAZ P., WORBE J. F., 1977. Transfert des acides gras volatils dans la paroi du cæcum isolé de rat. C. R. Soc. Biol., 171, 375-380.

PENNINGTON R. J., SUTHERLAND T. M., 1956. The metabolism of short-chain fatty acids in the sheep. The pathway of propionate metabolism in rumen epithelial tissue. Biochem. J., 63, 618-628.

SHERRATT H. S. A., 1968. The metabolism of the small intestine. Oxygen uptake and L-lactate production along the length of the small intestine of the rat and guinea-pig. Comp. Biochem. Physiol 24, 745-761.

SMITH R. M., OSBORNE-WHITE W. S., 1971. Synthesis of phosphoenol-pyruvate from propionate in sheep liver. Biochem. J., 124, 867-876.

SPENCER R. P., KNOX W. E., 1960. Comparative enzyme apparatus of the gut mucosa. Fed. Proc., 19, 886-897.

SPHAR S. L., KESSLER E. M., FLIPSER J., 1965. Utilization of blood acetate and butyrate by the isolated perfused goat rumen. J. Dairy Sci., 48, 228-233.

SRIVASTAVA L., HÜBSCHER G., 1966. Glucose metabolism in the mucosa of the small intestine. Biochem. J., 101, 48-55.

STEVENS C. E., 1970. Fatty acid transport through the rumen epithelium, 101-112. In PHILLIP. SON A. T. Physiology of digestion and metabolism in the ruminants. Oriel Press, Newcastle.

STEVENS C. E., STETTLER B. K., 1967. Transport of fatty acids mixtures across rumen epithelium. Am. J. Physiol., 213, 1335-1339.

TEJWANI G., KAUR J., ANANTHANARAYANAN M., RAMAIAH A., 1974. Concentrations of various effectors and substrates et phosphofructokinase in the jejunum of rat and their relation to the lack of Pasteur effect in this fissue. Biochem. biophys. Acta, 370,120-129.

WEEKES T. E. C., 1974. The in vitro metabolism of propionate and glucose by the rumen epithelium. Comp. Biochem. Physiol, 49, 393-406.

WEIGAND E., YOUNG J. W., McGILLARD A. D., 1972. Extent of propionate metabolism during absorption from the bovine reticulum. Biochem. J., 126, 201-209. 
WILSON T. H., WISEMAN G., 1954. The use of everted small intestine for the study of transference of substances from the mucosal to the serosal surface. J. Physiol., Lond., 123, 116-125.

WORBE J. F., MOTTAZ P., SADEGHI A., 1969. Influence de l'acide succinique et de l'acide $\alpha$-cetoglułarique sur l'activité métabolique de l'intestin grêle du rat. C. R. Soc. Biol., 163, 431-436.

WORBE J. F., MOTTAZ P., 1970. Métabolisme et absorption de l'acide butyrique par le cæcum de rat. J. Physiol. 62, 465-466.

WORBE J. F., MOTTAZ P., RICHARDOT D., 1971. L'absorption du glucose par la paroi du cæcum de rat. C. R. Soc. Biol. 165, 1066-1070. 